\title{
Constitutive Model of Shape Memory Alloy and its Finite Element Implementation for Medical Applications
}

\author{
Yanping Wang ${ }^{1, a^{*}}$ \\ ${ }^{1}$ College of Medicine, Xi'an International University, Xi'an, Shaanxi, China \\ a375031254@qq.com
}

Keywords: Shape memory alloys; Superelasticity; Constitutive law

\begin{abstract}
Shape memory alloy (SMA) have presented excellent behaviour for medical applications such as bio-compatible, high corrosion resistance, non-magnetic, and the unique physical properties, which replicate those of human tissues and bones. Based on the experimental results of super-elastic NiTi alloy, a three-dimensional super-elastic shape memory alloy (SMA) constitutive model for stress-induced martensite transformation is constructed in a framework of general inelasticity. In the proposed model, transformation hardening, reverse transformation of stress-induced martensite, elastic mismatch between the austenite and martensite phases, and temperature-dependence of transformation stress and elastic modulus of each phase are considered. The validity of such implementation was finally presented and compared with experimental data taken from the literature.
\end{abstract}

\section{Introduction}

SMAs are used in medical applications including stents, endodontics, sutures, medical tweezers, anchors for attaching tendon to bone, implants, aneurism treatments, eyeglass frames and guide wires and other medical devices and equipments in many fields including neurology, orthopaedics, cardiology and interventional radiology [1].

Some works are carried out on the implementation of constitutive model of SMA into finite element software [2,4]. Auricchio [2] had been implemented a super-elastic constitutive model successfully into the finite element codes, such as ABAQUS and ANSYS. In this work, based on the framework of the generalized plasticity, a temperature-dependent three-dimensional phenomenological constitutive model was developed to describe the thermo-mechanical deformation of super-elastic NiTi alloy. The prediction capability of the proposed model was verified by comparing the simulated results with the experimental ones. Finally, numerical example of finite element calculation is given to verify the validity of the implementation.

Constitutive Model. Generalized plasticity is firstly employed by Lubliner and Auricchio [5] to describe the thermo-mechanical responses of shape memory alloys. It is a local internal variable theory of rate-independent inelastic behavior which is based primarily on the loading-unloading irreversibility. The general mathematical foundation provides the theory the ability to deal with the non-standard cases such as non-connected elastic domains. With infinitesimal strain assumption, the additively decomposition of the total strain $\overline{\boldsymbol{\varepsilon}}$ into an elastic strain $\overline{\boldsymbol{\varepsilon}}_{\mathrm{e}}$ and an inelastic strain $\overline{\boldsymbol{\varepsilon}}_{\text {in }}$ yields:

$$
\overline{\boldsymbol{\varepsilon}}=\overline{\boldsymbol{\varepsilon}}_{\mathrm{e}}+\overline{\boldsymbol{\varepsilon}}_{\text {in }}
$$

Based on the assumption of small deformation, the total inelastic strain $\overline{\boldsymbol{\varepsilon}}_{\text {in }}$ is the stress-induced martensitic transformation $\overline{\boldsymbol{\varepsilon}}_{\mathrm{t}}$. As a result, the total strain expression is:

$$
\overline{\boldsymbol{\varepsilon}}=\overline{\boldsymbol{\varepsilon}}_{\mathrm{e}}+\overline{\boldsymbol{\varepsilon}}_{\mathrm{in}}=\overline{\boldsymbol{\varepsilon}}_{\mathrm{e}}+\overline{\boldsymbol{\varepsilon}}_{\mathrm{t}}
$$

After discretizing of the equations, the expressions are as follows:

$$
\Delta \overline{\boldsymbol{\varepsilon}}_{\text {in }}=\Delta \overline{\boldsymbol{\varepsilon}}_{\mathrm{t}}
$$


$\Delta \overline{\boldsymbol{\varepsilon}}_{\mathrm{e}}=\Delta \overline{\boldsymbol{\varepsilon}}-\Delta \overline{\boldsymbol{\varepsilon}}_{\mathrm{t}}$

The stress-induced martensitic transformation and its reverse transformation which can be defined by the volume fraction of martensite as the internal variable $\bar{\xi}$. The elastic stress-strain relation can be then expressed as:

$$
\overline{\boldsymbol{\sigma}}=\overline{\boldsymbol{\sigma}}^{*}-\overline{\mathbf{D}}^{\mathrm{e}}(\bar{\xi}): \Delta \overline{\boldsymbol{\varepsilon}}_{\text {in }}
$$

where $\overline{\mathbf{D}}^{\mathrm{e}}(\bar{\xi})$ is the equivalent elastic tensor [6,8], and $\overline{\mathbf{D}}^{\mathrm{e}}(\bar{\xi})=\left[(1-\bar{\xi}) \overline{\mathbf{D}}_{A}^{-1}+\bar{\xi} \overline{\mathbf{D}}_{M}^{-1}\right]^{-1}$.

where $\overline{\mathbf{D}}_{A}$ and $\overline{\mathbf{D}}_{M}$ are the elastic tensor of austenite and martensite, respectively. Considering the deviatoric stress of the above equation, it can be gotten $\overline{\mathbf{D}}^{\mathrm{e}}: \Delta \overline{\boldsymbol{\varepsilon}}_{\text {in }}=2 \bar{G} \Delta \overline{\boldsymbol{\varepsilon}}_{\text {in }}$, and:

$$
\overline{\mathbf{S}}=\overline{\mathbf{S}}^{*}-2 \bar{G} \Delta \overline{\boldsymbol{\varepsilon}}_{\text {in }}
$$

where $\bar{G}$ is theshear elastic modulus, and $\overline{\mathbf{S}}^{*}$ is the deviatoric stress of $\overline{\boldsymbol{\sigma}}^{*}$.

As mentioned in the references[9, 10], some phase transformations are pressure-dependent. To model such an effect, the Von-Mises-typed transformation surfaces are introduced:

$$
\bar{F}(\overline{\boldsymbol{\sigma}}, \bar{q})=\bar{\sigma}_{\mathrm{eq}}-\bar{\sigma}_{\mathrm{y}}(\bar{q})=0
$$

where $\bar{\sigma}_{\text {eq }}=\left[\frac{3}{2} \overline{\mathbf{S}}: \overline{\mathbf{s}}\right]^{\frac{1}{2}}$ is the von Mises equivalent stress. The phase transformation to describe the forward transformation and its reverse transformation are introduced as follows:

$$
\begin{aligned}
& \bar{F}_{\mathrm{AM}}(\overline{\boldsymbol{\sigma}}, \bar{\xi})=\bar{\sigma}_{\mathrm{eq}}-\sigma_{s, T}^{\mathrm{AM}}(\bar{\xi})=0, \text { forward transformation } \\
& \bar{F}_{\mathrm{MA}}(\overline{\boldsymbol{\sigma}}, \bar{\xi})=\bar{\sigma}_{\mathrm{eq}}-\sigma_{s, T}^{\mathrm{MA}}(\bar{\xi})=0, \text { reverse transformation }
\end{aligned}
$$

here, $\sigma_{s, T}^{\mathrm{AM}}$ and $\sigma_{s, T}^{\mathrm{MA}}$ are the start stresses of the forward transformation and the reverse transformation, respectively.

In this paper, the reversible martensite volume fraction $\bar{\xi}$ can be related to the transformation strain $\bar{\varepsilon}_{\mathrm{t}}$ by the following formula:

$$
\bar{\xi}=\bar{\varepsilon}_{\mathrm{t}} / \varepsilon_{\mathrm{L}}
$$

where, $\varepsilon_{\mathrm{L}}$ is the maximum phase transformation strain under uniaxial tension, which can be determined by the experimental test under loading and unloading.

Similar to the classical plasticity, the transformation strain rates obey the normality rule, i.e., they are normal to the transformation surface in the stress space as

$$
\begin{aligned}
\Delta \boldsymbol{\varepsilon}_{\mathrm{t}}^{\mathrm{GM}} & =\sqrt{\frac{3}{2}} \varepsilon_{\mathrm{L}} \Delta \bar{\xi} \overline{\mathbf{n}}_{\mathrm{AM}}, \Delta \bar{\xi}>0, \text { forward phase transformation } \\
\Delta \boldsymbol{\varepsilon}_{\mathrm{t}}^{\mathrm{GA}} & =\sqrt{\frac{3}{2}} \varepsilon_{\mathrm{L}} \Delta \bar{\xi} \overline{\mathbf{n}}_{\mathrm{MA}}, \Delta \bar{\xi}<0, \text { reverse phase transformation }
\end{aligned}
$$

with

$$
\overline{\mathbf{n}}_{\mathrm{AM}}=\frac{\bar{\partial} \bar{F}_{\mathrm{AM}}(\overline{\boldsymbol{\sigma}}, \bar{\xi})}{\bar{\partial} \overline{\boldsymbol{\sigma}}}=\sqrt{\frac{3}{2}} \frac{\overline{\mathbf{s}}}{\bar{\sigma}_{\mathrm{eq}}}
$$




$$
\overline{\mathbf{n}}_{\mathrm{MA}}=\frac{\bar{\partial} \bar{F}_{\mathrm{MA}}(\overline{\boldsymbol{\sigma}}, \bar{\xi})}{\bar{\partial} \overline{\boldsymbol{\sigma}}}=\sqrt{\frac{3}{2}} \frac{\overline{\mathbf{s}}_{\overline{\mathrm{s}}}^{\bar{\sigma}_{\mathrm{eq}}}}{}
$$

In this paper, the phase transformation, austenite yield and martensite yield behavior under different temperature are considered in the process of implicit stress integral solution. In general, it is assumed that the stress-induced martensitic transformation, and there is no interaction between them. The isotropic elastic-plastic constitutive model with implicit stress integration method is extended to that method of the constitutive modeling of SMA. According to the above assumptions, Eq. (6) can be further expressed as follows for the implicit stress integration process of the phase change behavior:

$$
\overline{\mathbf{s}}=\overline{\mathbf{S}}^{*}-2 \bar{G} \Delta \overline{\boldsymbol{\varepsilon}}_{\mathrm{t}}
$$

By Eqs. (16a-b), it can be obtained:

$\Delta \overline{\boldsymbol{\varepsilon}}_{\mathrm{t}}^{-\mathrm{MA}}=\sqrt{\frac{3}{2}} \varepsilon_{\mathrm{L}} \Delta \bar{\xi}_{\overline{\mathbf{n}}}^{-} \overline{\mathbf{m}}_{\mathrm{MA}}, \Delta \bar{\xi}>0$, forward phase transformation

$$
\Delta \overline{\boldsymbol{\varepsilon}}_{\mathrm{t}}^{-\mathrm{MA}}=\sqrt{\frac{3}{2}} \varepsilon_{\mathrm{L}} \Delta \bar{\xi}_{\overline{\mathbf{n}}} \overline{\mathrm{MA}}_{\mathrm{A}}, \Delta \bar{\xi}<0, \text { reverse phase transformation }
$$

in which, $\overline{\mathbf{n}}_{\mathrm{AM}}=\overline{\mathbf{n}}_{\mathrm{MA}}=\overline{\mathbf{n}}_{\mathrm{t}}=\sqrt{\frac{3}{2}} \frac{\overline{\mathbf{s}}}{\bar{\sigma}_{\mathrm{eq}}}$

Verification of Proposed Model. The results of the phase transformation simulations by the proposed model are compared with the experimental data by Kang [11]. To verify the proposed model, the super-elastic stress-strain curves at die rent temperatures are simulated using the parameters listed as follows to identify the parameters.

$E_{\mathrm{A}}^{T_{0}}=41.0 \mathrm{GPa}, E_{\mathrm{M}}^{T_{0}}=37.0 \mathrm{GPa} ; v_{A}=v_{M}=0.33 ; C_{A M}=8.0 \mathrm{MPa} / \mathrm{K}, C_{M A}=8.8 \mathrm{MPa} / \mathrm{K} ; k=0.16 ; \sigma_{s, T 0}^{A M}$ $=353.0 \mathrm{MPa}, \sigma_{f, T 0}^{A M}=381.0 \mathrm{MPa}, \sigma_{s, T 0}^{M A}=141.0 \mathrm{MPa}, \sigma_{f, T 0}^{M A}=122.0 \mathrm{MPa} ; \varepsilon_{L}=0.035 ; \quad T_{0}=295 \mathrm{~K} ; h_{\mathrm{M}}^{\mathrm{p}}=$ 6.7GPa.

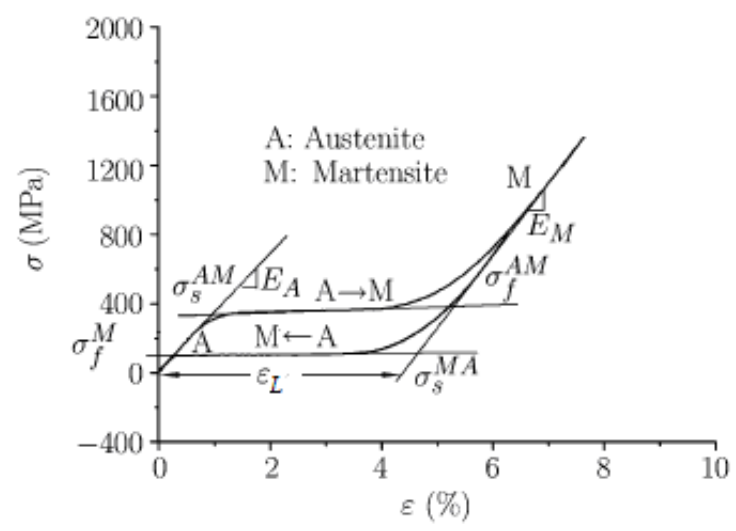

Figure 1. SMA phase transformation and plasticity behavior diagram 


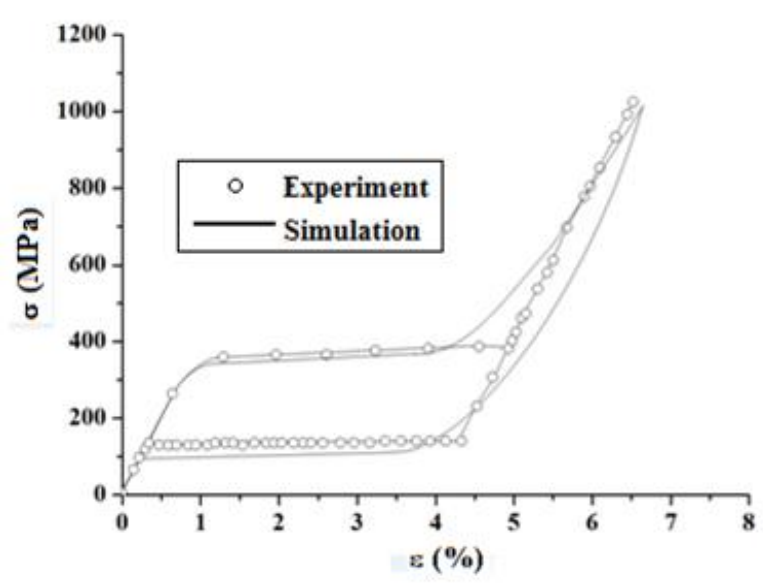

Figure 2. Uniaxial tension and unloading of NiTi shape memory alloy with phase transformation

The uniaxial tension and unloading case of the material is calculated based on the proposed model. The simulation results are shown in Fig.2. It can be seen from Fig.2 that the stress-induced martensitic transformation occurs. As the load is lower than the phase transformation finish stress $\sigma_{f, T}^{M A}$, the austenite will take place elastic unloading as following. That elastic unloading curve is coincident with the initial elastic loading one. It can be seen from Fig. 2 that the response peak stress is about $1000 \mathrm{MPa}$, and the martensite can still be completely transformed into austenite when unloading. The simulated results show good agreement with the experimental ones. The results show that the proposed constitutive model can predict the thermodynamic behavior in the super-elastic NiTi alloy. It can be predicted that the model can also give a reasonable prediction results for other working conditions and other experimental results in the temperature range.

\section{References}

[1] Jani J.M., Leary M., Subic A. and Gibson M. A. A review of shape memory alloy research, applications and opportunities, Mater Design. Vol. 56 (2014), p.1078-1113.

[2] F. Auricchio and R.L. Taylor, Shape-memory alloy modeling and numerical simulations of the finite-strain superelastic behavior, Comput Method Appl Mech Eng. Vol. 143 (1997), p.175-194.

[3] F. Auricchio, A robust integration algorithm for a finite strain shape memory alloy superelastic model, Int. J. Plasticity. Vol. 17 (2001), p.971-990.

[4] N. Rebelo, M. Hsu and H. Foadian, Simulation of super-elastic alloys behavior with abaqus. Proc. International Conference on Shape memory and Super-elastic Technologies. SMST, 2000, Pacific Grove (USA, 2001) 457-469.

[5] L. Saint-Sulpice, S.A. Chirani, S Calloch. A 3D superelastic model for shape memory alloys taking into account progressive strain under cyclic loadings, Mech. Mater. Vol. 41 (2009), p.12-26.

[6] Ivshin Y., Pence T. A thermomechanical model for a one variant shape memory material, J. Intel Mat. Syst Str. Vol. 5 (1994), p.455-473.

[7] Auricchio F., Marfia S., Sacco E. Modelling of SMA materials: Training and two way memory effects, Comp. Struct. Vol. 81 (2003), p.2301-2317.

[8] Zaki W., Moumni Z. A 3-D model of the cyclic thermomechanical behavior of shape memory alloys, J Mech. Phys. Solids. Vol. 55 (2007), p.2427-2454.

[9] Liang C., Rogers C. One-dimensional thermomechanical constitutive relations for shape memory materials. J. Intel Mat. Syst Str. Vol. 1 (1990), p.207-234.

[10] Auricchio F., Sacco E. A one-dimensional model for superelastic shape memory alloys with diffeent 
elastic properties between austenite and martensite, Int. J Nonlin. Mech. Vol. 32 (1997), p.1101-1114.

[11] Kang G.Z., Kan Q.H., Qian L.M. and Liu Y.J. Ratchetting deformation of super-elastic and shape-memory NiTi alloys, Mech. Mater. Vol. 41 (2009), p.139-153. 